$\Rightarrow$ CELL DEATH

\title{
Fatal cut
}

DOI:

10.1038/nrm2020

URLs

ATG5

http://ca.expasy.org/uniprot/

Q9H1Y0

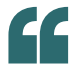

...this finding

has clearly

important

implications

for anti-cancer

therapies.
Autophagic cell death is thought to be a fail-safe mechanism of suicide that the cell switches to under conditions in which apoptosis does not work. A new study from the group of Hans-Uwe Simon indicates that the reverse situation can also occur - the cell can switch the autophagic system towards apoptosis.

Simon and colleagues noticed that overexpression of the autophagyrelated gene-5 (ATG5) protein increased the cell's susceptibility to undergo apoptosis in response to cell-death stimuli, including certain anti-cancer drugs. By contrast, ATG5-deficient cells showed a strongly reduced sensitivity towards anti cancer agents.

When Simon and co-workers investigated a possible role for ATG5

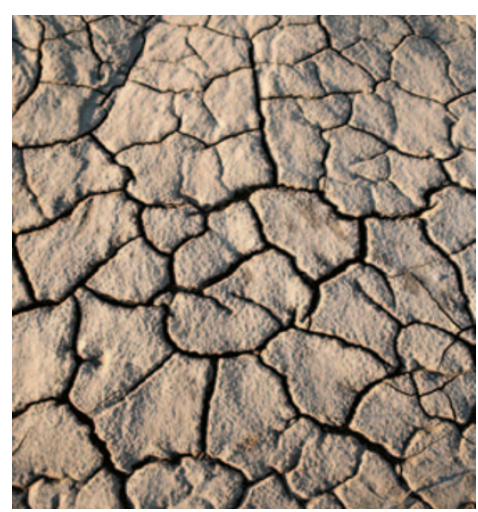

in apoptosis by using a model of spontaneous neutrophil apoptosis, they detected a truncated ATG5 product in apoptotic, but not in nonapoptotic, neutrophils. They suggested that ATG5 might be a target of proteases that are activated during apoptosis, and identified calpain-1 and -2 as ATG5-specific proteases. Indeed, incubation of a calpain inhibitor with neutrophils prevented both apoptosis and ATG5 cleavage. Overexpression of an ATG5 mutant that lacked the calpain cleavage site in ATG5-deficient cells did not increase sensitivity to anti cancer drugs. The authors therefore concluded that calpain-mediated cleavage of ATG5 is a crucial pro-apoptotic event. In addition, the authors found that autophagic activity is not required for the apoptotic effect of truncated ATG5.

Simon and colleagues showed that truncated ATG5 induced cytochrome $c$ release and apoptosis in cells that were not protected by high levels of the anti-apoptotic protein BCL2. ATG5 colocalized with a mitochondrial marker, and the appearance of truncated ATG5, but not of fulllength ATG5, in the mitochondrial fraction coincided with cytochrome $c$ release and caspase- 3 cleavage. The authors also showed that truncated
ATG5-mediated apoptosis could be blocked by a caspase inhibitor.

But how does truncated ATG5 trigger cytochrome $c$ release? The authors proposed that truncated ATG5 might bind to BCL2-family members. They found that fulllength and truncated ATG5 did not bind to BAX; however, only truncated ATG5 binds to BCL- $\mathrm{X}_{\mathrm{L}}$ in apoptotic, but not in non-apoptotic, cells. The authors speculated that truncated ATG5 promotes apoptosis by blocking the function of the anti-apoptotic 'survival' protein BCL-X ${ }_{L}$, which leads to BAX and/or BAK activation. Although this hypothesis requires further testing, it is supported by the observation that ATG5-induced cell death was blocked by high levels of BCL2.

Although the details of this molecular link between autophagy and apoptosis need to be worked out further, this finding has clearly important implications for anti cancer therapies.

Arianne Heinrichs, Chief Editor,

Nature Reviews Molecular Cell Biology

ORIGINAL RESEARCH PAPER Yousefi, S. et al. Calpain-mediated cleavage of Atg 5 switches autophagy to apoptosis. Nature Cell Biol. 8, 1124-1132 (2006) FURTHER READING Codogno, P. \& Meijer, A. J. Atg5: more than an autophagy factor. Nature Cell Biol. 8, 1045-1047 (2006) 